\title{
Complete nucleotide sequence of the genome of an apple isolate of apple chlorotic leaf spot virus
}

\author{
K. Sato, N. Yoshikawa* and T. Takahashi \\ Plant Pathology Laboratory, Faculty of Agriculture, Iwate University, Ueda 3-chome, Morioka 020, Japan
}

The complete nucleotide sequence of the genome of an apple isolate of apple chlorotic leaf spot virus (ACLSVA) was determined. The genome is 7552 nucleotides excluding the poly(A) tail and contains three open reading frames (ORFs 1,2 and 3 ), encoding proteins with $M_{\mathrm{r}}$ values of $216503(216.5 \mathrm{~K}), 50453(50 \cdot 4 \mathrm{~K})$ and 21394 (21.4K), respectively. Nucleotide sequence comparisons between ACLSV-A and the previously sequenced ACLSV from plum (ACLSV-P) showed that the sequence identity at the nucleotide level was $79.8 \%$. Amino acid sequence identities of ORFs 1 and 2 between both isolates were $88.4 \%$ and $79.9 \%$, respectively. The $21.4 \mathrm{~K}$ protein encoded by ORF 3 of ACLSV-A had an amino acid sequence identity of $88.6 \%$ with the $28.3 \mathrm{~K}$ protein encoded by ORF 3 of ACLSV-P. Immunoblot analysis of the $21.4 \mathrm{~K}$ protein expressed in Escherichia coli showed that this protein is the coat protein of ACLSV-A.
Apple chlorotic leaf spot virus (ACLSV) is distributed worldwide in fruit trees including apple, peach, pear, plum, cherry and apricot (Lister, 1970; Németh, 1986). In Japan, this virus is one of the causative agents of apple topworking disease and induces lethal decline in apple trees grown on Maruba kaido (Malus prunifolia ringo) rootstocks (Yanase, 1974). ACLSV has very flexuous filamentous particles, approximately 600 to $700 \mathrm{~nm}$ in length and contains a polyadenylated plus-sense, ssRNA with an $M_{\mathrm{r}}$ of $2.48 \times 10^{6}$ and a single coat protein of $22 \mathrm{~K}$ (Yoshikawa \& Takahashi, 1988). At present, this virus is classified into the closterovirus group based on its particle structure (Francki et al., 1991). Recently, German et al. (1990) reported the nucleotide sequence of the genome of ACLSV isolated from plum (Prunus domestica) (ACLSV-P) in France.

In this paper, we report the complete nucleotide sequence of the genome of ACLSV isolated from apple (ACLSV-A) in Japan and the comparisons of the nucleotide sequence and the deduced amino acid sequence with those of ACLSV-P.

ACLSV-A (isolate P-205), originally isolated from an apple tree (Yanase, 1974), was propagated in Chenopodium quinoa and purified as described previously (Yoshikawa \& Takahashi, 1988). Viral RNA was extracted from purified virus by dissociation in $2 \%$ SDS

The nucleotide sequence reported in this paper will appear in the DDBJ, EMBL and GenBank nucleotide sequence databases under the accession number D14996. for $15 \mathrm{~min}$, followed by extraction with phenolchloroform, precipitated from $70 \%$ ethanol and suspended in water. First and second strand cDNAs were prepared from $2 \mu \mathrm{g}$ of ACLSV-A RNA according to Gubler \& Hoffmann (1983) using oligo(dT) or random hexanucleotides as primers. The dsDNAs were bluntended using T4 DNA polymerase and ligated into the EcoRV site of Bluescript $\mathrm{KS}^{+}(\mathrm{BS})$ or dC-tailed and annealed to dG-tailed, Pst $\mathrm{I}$-cut pUC9 (Yoshikawa et al., 1988). The resulting plasmids were used to transform competent Escherichia coli DH5 $\alpha$ cells.

The cDNA clones or subclones produced after the ligation of restriction fragments into BS were digested with exonuclease III and mung bean nuclease (Henikoff, 1984) and the resulting deletion mutants were used for sequencing by the dideoxynucleotide chain termination method (Sanger et al., 1977) using Sequenase version 2.0 (United States Biochemicals). Each base was determined by sequencing at least two independent clones. More than $80 \%$ of the genome was sequenced in both directions and dITP was used to resolve ambiguities resulting from compression.

The sequence of the 5'-terminal region of ACLSV-A RNA was determined by extending a synthetic primer (5' AGGCGTTACGTCAATCTG 3') complementary to nucleotides (nt) 38 to 55 of the viral RNA using reverse transcriptase and terminal deoxynucleotidyl transferase (Deborde et al., 1986). All nucleotide sequence data were collected and analysed using the program GENETYX version 8.0 (Software Development Co. Ltd).

The genome of ACLSV-A consists of $7552 \mathrm{nt}$ ex- 


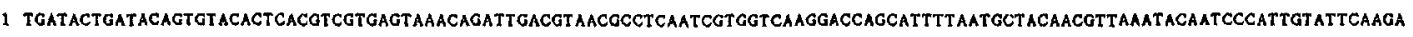
121 ACTTTCCGTTTAACTACGTGAAAGGTTACAAATGGCTTTCTCTTATAGAACGCCACAGGAAGAACTTCTGAGCAGGTTACCTCAGTCTCAGCAGGAAGCGATCAGCGGATTTCAGTACGA ORF1 $M$ A $F$ S Y B T P Q E E L L S R L P Q S Q Q E A I S G F Q Y E 242 GCGATTTCAAAAGGAGGAAGAGAAGAAGGTGGAAAACTTCTCTTTTTACCTACCGOAGAAGACCAGGGAGTGGTTCACTAAGTCAGGTGTATATCTCTCACCCTTTGCTTATGTCAATCA 361 TTCTCACCCTGGTTGCAAAACTCTGGAGAACCACCTTTTATTTAATGTTGTAGCT AGTTATATTAGTAAGTATTCTTATGTAGCTTGTCTTAGTATTAaGTCGAATAAAATGAGCAAGAT 481 GGAACGTTTGGGTTCCAATTCAGTCAAAACCTATGACATTTTGAACAGATT AGTTACTGCTAAGGACAAGGCTCGATATGGACCTTTGGCCAGGCCTGAGCGGTCTCCATGTCCAAAGAA E R L G S N S V K T Y D I L N R L Y T A K D K A R Y G P L A R P B R P C P B K 601 AACANACATTTTCATTCATGATGAGATCCATTATTGGTCAAGGGATCAACTTGAGACATTCCTTCAGGTTCACAGACCCAAAAATTTGTGGGCGACTCTTGTTTTTCCCCCTGAGATCT

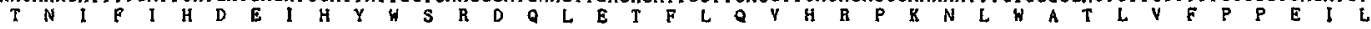
721 GGCTGGTTATAAGTCGTCAGTTTTACCATTTCTTTACCAGTTTGAGATTCATGGAaAaGATTTGGTTTACATGCCTGATGGaGTTAGATCAGAGAGTTACACTCAACCTCTTGAGAATGG

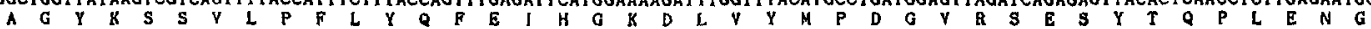
84L ATTTCTTCTGAGT TCAAGCAGTATACTTGTGAGAaACAaGGCTACTGGaGTTGAAATTAGATATCAGGTTAGTCTTGTGTATTCTCTGGOTTCTCACCATTTGTTCCACATTTACCCCAC 961 GGAGGACCTGATGAAAGAAGAGGTGAGAAGGTTTGGACCTTATCATTTGTTCGATGTGGGTCATTATTTGTCAAGCCAGTGCGCGTCCCTATCCAAGACTTCCCTTTGAGTGTCTTCAA 1081 AAAGATATTCATTTATCTTAGTTCATTAAAGAAGCCTGATGTTCAGTCTGCAGTGGCCAAACTCAGACAGTTATCCGATGCCGACATTTCCATTGAGAGTGTTTTCATOGTGCAGGAGTT

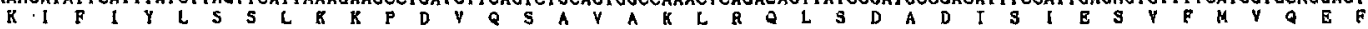
1201 TGCAAGTAGGGTGGAAAAAATGGAGTCGOAAGTTGGTCCTGCTCATTTTGGAGTGCATGAMGGACTGOTTTTTTGATAAGCTGCCATACAGAGAGGTGCTGGAAAAATTGOATTGGC

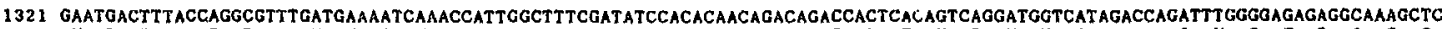

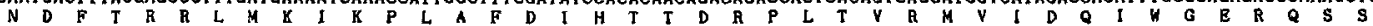

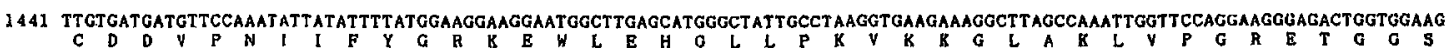
1561 TGATTACCCAGAMGAAATCTATTCTGATTTGCTGTCATCCACCTCAATTTGGAGGTCTTATGATGAGAATCTAAGGCACAGGAAAGCCAGTCCTATTGTGATACTTAAGTCCGAAAAGGC D Y P E E I Y S D L L S S T S I W R S Y D E N L R H R 1681 ATACTCTGAGGCCCCTGGGTTCAGCTCAAACTGTATCAGCCTGTGTTCTACGCCTTTTGGTGAGGTTATTGAAAGGACCCCTTTTGAGGTGGAAGGGAAAGGAAGAAAAGAGAGTTGTC

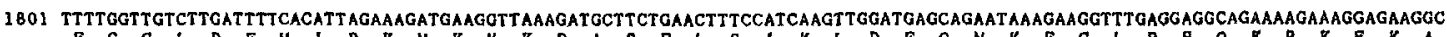

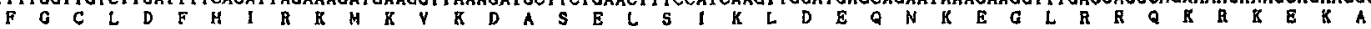

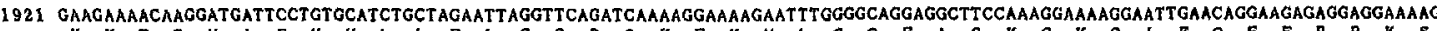

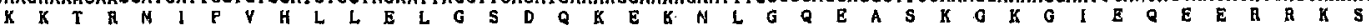
2041 TGATGAAGCAAAGTTGATTCTGGGCCTCOGGAGTTTGCAGCATAAAAGCGGAAAATCCTGTTGATGCACAACACATTGCTGAGCCCGTGCCATGTTTGAAATTGAATGATCTGATTGG $216 \mathrm{~L}$ CAAAGAGAAAATCTGTTCTTCTGGTCTT ATCAAAACAGTTGGAATGATTATCTCACTTTGGCCAGGCAGATAGAGGATATGCCCCTCGCCCAGTTGAAGAACAGGAAAGCAGCATATTT 2281 CTGCATTGACTACCCTATGGTGTACTTTCATGACAAGATTTCATACCCAACTTTTGAGGTTACTGGTGAGATCAAACAAATCATCATGAGAGCAAGAGACAAATGGGGTGCAAATTTCAA C I D Y P M V Y F H D B I S Y P T F E A T G E I K Q I I M R A R D K W G A

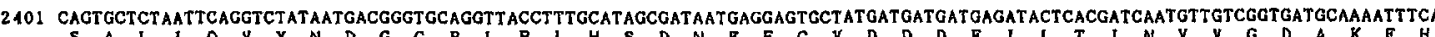

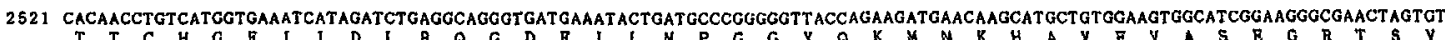

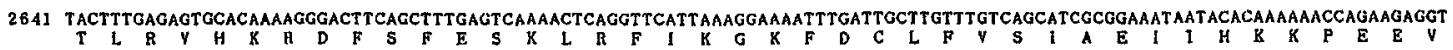

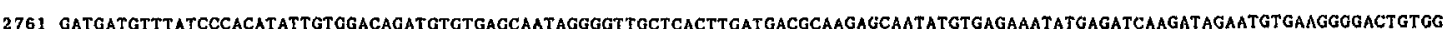

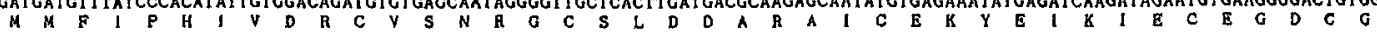
2881 CCTAGTTGAGTGTGGAACAATTGGTCTGTCTGTTGGGAGGATGCTCCTCAGAGGGAATCATTTTACAGTGGCTTCGGTAAGAAGATCATCCATGGACTCCCTTGCCAATTCATCAAAAGA

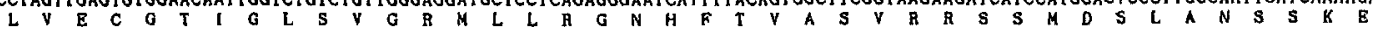
3001 ACTCAAATCAAATGGAGTCTTGGATCATGTAATGTTTAATTTTCATAAAAGACTTAGATCCGTGGAACCTGATCTGACTGT AGAGGAAATCAAAGTGGTTCTTCAAGAGCGGGGAAGTT $L K$ S N G V L D H V M F N F

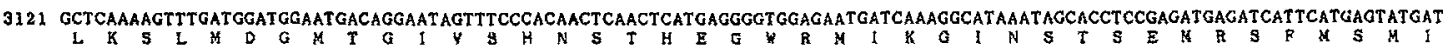
3241 CAAAGGTAAAAGTGAAGAGACCAGGGGTGACTTATTTOACAGAGTTCAGGAACTGAACTTCATGAAGOTT AAGATCTACGGCATATTTGGGTTTGCTGGATCAGGGAAGAGTCATGCAAT $K G K$ K E E T R G D L F D R V G E L N F M K V K I Y G I F G F A G S G K S H A I 3361 TCAAAATCTCATTCAGACAGAATTCAAACGATCACAAGGTGTTATGGTGATCTGTCCAAGAAGGTTTTTGGCCAAGGACTGGAGTGAAAAAGGGGTGGATGAGAAGGATATTAAAACCTT 3481 TGAGAGCGCACTGAGTCAGATGTTAAAGGAAAGAGATTATTCATACTTGATGAAATCAGTTTGTTOCCTAAAGGTTTCACTGACCTCTTGATGCTCAAAATGCATATGGAaGGGATTCT 3601 GAAAAAGTCAACAATTGTGTGTATTGGTGACCCTCTGCAAGCTGGGTACTTCTGTCCTAAGGATGACAATTATTTGTCTAGGGAGGGAGAAATCAAGAGGCTTTTCAAAGATGGGGTCAA

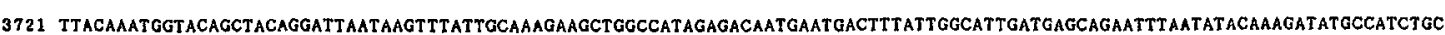

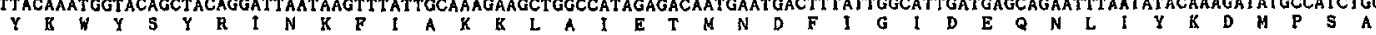
3841 TCACCATTTCATGGAGAACAAAGGGAATCACATTGAaGTGATTCTCGTTCCCAGCATGAT AGAGAAAGAGTTATACTCGAATTACGGCAATGTGATGACTTTTGGCGAGTCACAGGGTCT H H F M E N K G N H I E Y I L V F S M I E K E L Y S N Y G N Y M T F G E S G G L

3961 TACTTTCAACTOTGGGGTCATAGTGCTGTCCGAagagGCCAAGCTATGCTCCGATGCTCATATAATOGTGGCGATCACAaGaTTTAGGCGAGGTTTCTGCTTTGCACTTGGCAGTAAAGG 4081 GTCAAAGGAGGATTACATOAGATCCATCAAAAGTGGATTGCTTCAAAGGATGTGCTCCGGGATTGGTGCTTCTAAGGAATTTATTCTAGGATCCTCTTCAGTCAAATTAATCCTGAGTGA

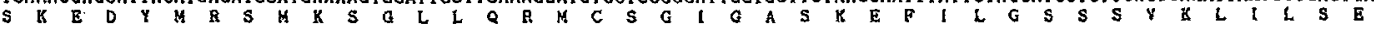

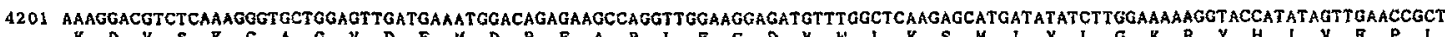

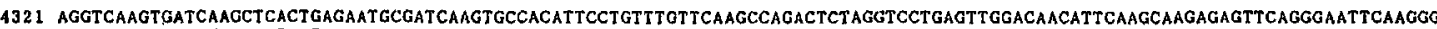

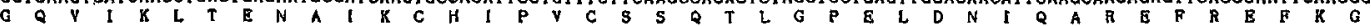

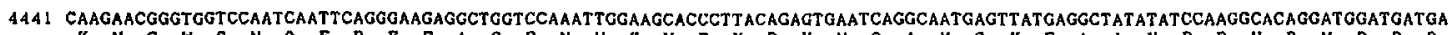

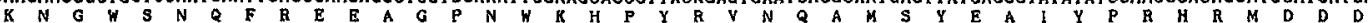
4561 TCTAACCTTTCTTGCTGCCATCAAGAAGAGGCTTCGTTTCGATAACGTTGCTAATAATTATGCAAAGTTTAAAGCAGCAGAGAGTAGAGGCAGGTAGTT AGCAAAAGTTTTCTTGCG ACA 


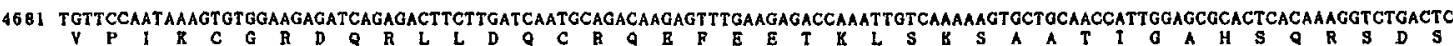
4801 TGATTGGCCATTGGACAAAATCTTTCTTTTCATGAAGTCACAATTATCCACCAAGTTCGACAAGAGGTTCACAGAAGCCAAAGCQGGTCAGACTTTGGCCTGCTTTCCGCATAAGATACT 4921 TGTTGAGTTCAGCCCATGGTGCAGGTACACAGAGAAAGTACTCACTGCAAACCTTCCAGATAATTATTACATTCATCAAAGGAAGAACTTCAGTGAACTTGAAGATTTYGCAAAGAGGT

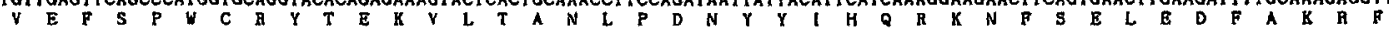

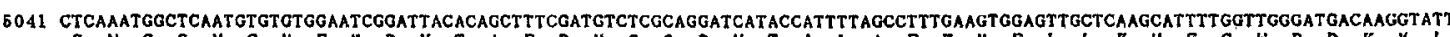
$S$ N G S M C Y E S D Y T A F D Y S Q D H T T L A F E Y E L L R H F G W D D K V L

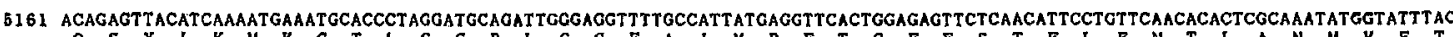

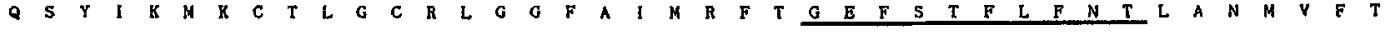

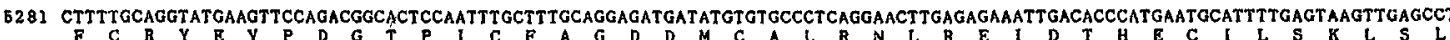
6401 CAAAGCAAAAGTGAACAGAACAAACGT ACCGATGTTTTGTGGATGGAGGTTGTGTTTCGACGGACTTATAAAGGAACCTTGTTTGATTTACGAAAGATTGCAAGTTGCAATTGAAAATGO

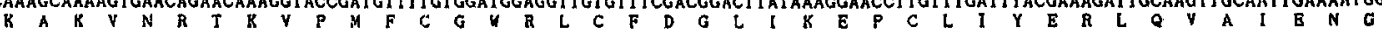

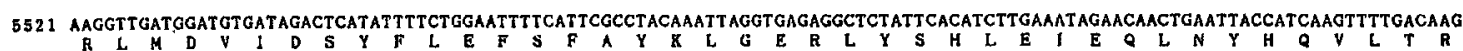

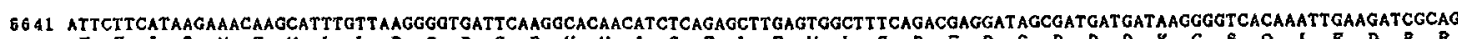

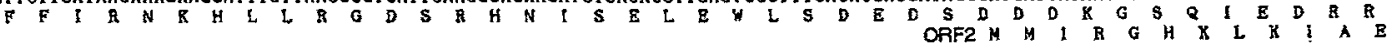

5761 AAGGGGATATTCCAATTGCTGGGGTGAAAGCTCCAGAATTTATTCTGATGT GAGTCCTTTCAGGAAGGCCTCTGACGTTATGATTCATTGGAATGAATTCGTATTCAAGGTTATGCCAO

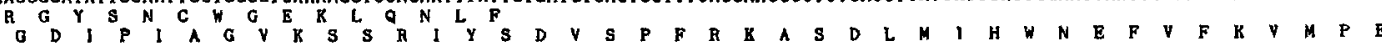

8881 AGOATATTGCAGGTGATGGTTTTAGATTGGCATCCATACCTOTTATACCTTCCTCAGAGGTTCAAGCGGTOCTGAGAAGAGGGAGAGTACCAACTACGTUCATTGGGGAGCACTATCAA D I A G $D$ G

6001 TATCCATTGACGCACTGTTCAGAAAGAATGCCGGGGTTTCAGGGTGGTGTT ATGTGTACGACAACAGATGGGAAACTTTTGAGCAGGCGATGCTACAAAAGTTTCGGTTCAACCTGGATA

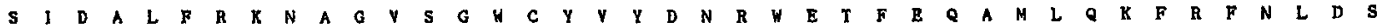

6121 GTGGCTCGGCCACATTGGTOACCTCACCGAATTTTCCAGTTTCATTGGATGATCCTGGTTTGTCCAATTCGATCAOTGTAGCGGTGATGTTTGAGAATCTGAACTTCAAGCT TGAAAGCT

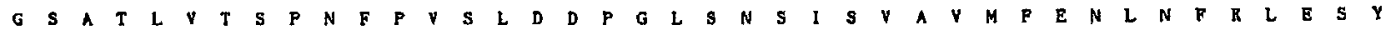

6241 ACCCTATAAGTGTTAGGGTAGGGACATGTGTAGATTCTTCGACAGCTTCTTGAGTTGCGTCAAGAATAAAGTGGACTCCAACTTTTTGCTTGAGGCCGCTAATGCAGATCCGCTTGGCG

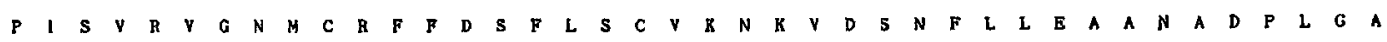
6361 CGGGTGCTTTCGGGTTTGAACAGOACOATCAGGTCTCTGAATTGTTCAATTATATTCAGACTGTCCCAACCCAGGCAATTAAATCCAGGGAGCATGAAGTACCGAGGGGTTTGTTCGGTA

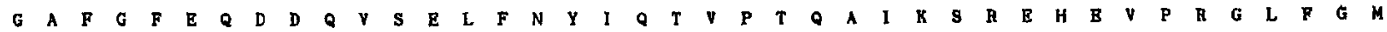

6481 TGATGGGAAAAAAGAAGgTCAAATCTTTTGAGTTTGCCTCTGGTTCAAGAAATCTGGGAAGAAGAAAACCGCAACGCGGAAGACCTTTGGAAAGATCTGCTAGCCTGAGAGTTGCTCCTG

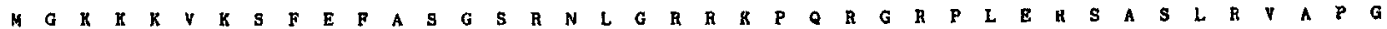
6601 GATTTAGGTCCCAGgaTGAGAGAGTTGAACATCAAGGATTATCGACTGACTCTGATTTTGAAAATTTTCTGAGGAAAGGCAAAGGAAAGCTGGAACTGAATCCATTACTTCAGAAGGGT

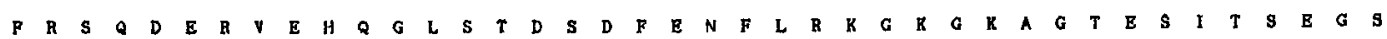
6721 CATCCTTCGATAACATTTCTGCAAGAGAUTTTCAGTTTGCTAGACAAGATCAGAAGCCGAAGGATGGCGGCAOTGCTGAACCTCCAATTAAAAGTGGACGCAGATCTGAAGCGTTCCTG

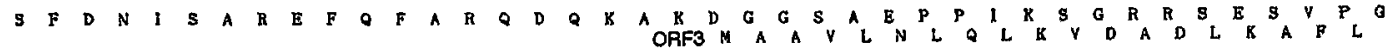
6841 GCCGCAGAaGgCAGACCCCTTCATGgAAAGACAGgGgCAATTCTGGAACAGACACTGGAGGCCATCTTCGCGAACATAGCGAT ACAGGGGACCTCGGAACAAACAGAGT TCCTGGACGTG

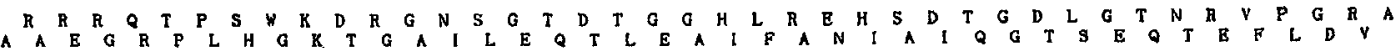
6961 CTGGTGGAGGTGAATCCATGGAGgATCAGAAGGTGGTGGGGTCATTCAATCTGAAGGAGGTAGTCGGTTTGATCAAAATATTCAGGACTACATCTTCGGACCCGAATATAAGCAGCATG

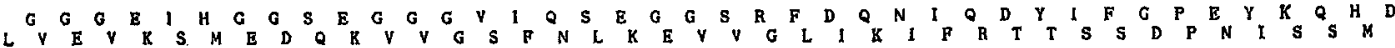
$70 B 1$ ACCTTCCGCCAAGTGTGTGAGGCATTTGCCCCGGAGGCAAGAAATGGGCTCGTAAAGCTAAAGTACAAAGGGGTCTTCACAAACCTCT ITTCTACTACGCCTGAAGTGGGGGGAAGTAT

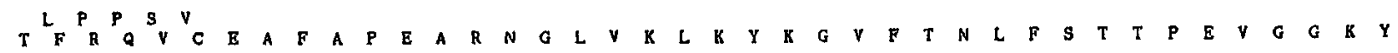
7201 CCGGAGCTCATGTTTGACTTCAATAAGGGCCTAAATATGTTCATAATGAACAAAGCCCAGCAGAAAGTGATAACTAATATGAACCGGCGTCTTTTACAGACTGAATTTGCAAAGAGCGAA

P E E L M 7321 AATGAGGCGAAATGTCGTCTGTTACAACTGATCTTTGCGTTAGTCTCATAAGGAGTTTGGTTTAATAAATAAAGTTAATAAATAGTGTGCATTTGGTTTAATGTCAAT TGTGTGTAT

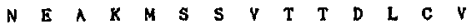

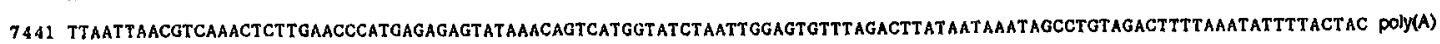

Fig. 1. The complete nucleotide sequence of the ACLSV-A genome and the amino acid sequences of three ORFs. The amino acid sequences of the conserved motifs associated with the helicase and the RNA polymerase are underlined.

cluding the $3^{\prime}$ poly(A) tail (Fig. 1), three nt fewer than that of ACLSV-P reported by German et al. (1990) (Table 1). Analysis of the putative open reading frames
(ORFs) of the nucleotide sequence in both positive and negative strands showed that the genome of ACLSV-A contains three ORFs in the positive strand (Fig. 1). 
Table 1. Comparisons between ACLSV-A and ACLSV$P$ genomes

\begin{tabular}{lrrr}
\hline & ACLSV-A & ACLSV-P & Identity \\
\hline Length excluding poly(A) tail & & & \\
Whole genome & $7552 \mathrm{nt}$ & $7555 \mathrm{nt}$ & $79 \cdot 8 \%$ \\
5' non-coding region & $151 \mathrm{nt}$ & $151 \mathrm{nt}$ & $92 \cdot 7 \%$ \\
3' non-coding region & $187 \mathrm{nt}$ & $190 \mathrm{nt}$ & $81 \cdot 2 \%$ \\
$M_{\mathrm{r}}$ of ORF products & & & \\
ORF 1 & 216503 & 216559 & $88 \cdot 4 \%$ \\
ORF 2 & 50453 & 50831 & $79 \cdot 9 \%$ \\
ORF 3 & 21394 & 28376 & $88.6 \%$ \\
\hline
\end{tabular}

\section{ACLSV-A(5715) A C GAGGAT A G C GATGA TGA T A A $* * * * * * * * * * * \quad * * * * * * *$ \\ ACISV-P(5712) A C GAAGATGG C GA CAA TGATAA}

Fig. 2. The nucleotide sequences surrounding the ORF 2 initiation codons of ACLSV-A and ACLSV-P. Identical nucleotides are shown by asterisks. Second ATG codons within ORF 2 are underlined.

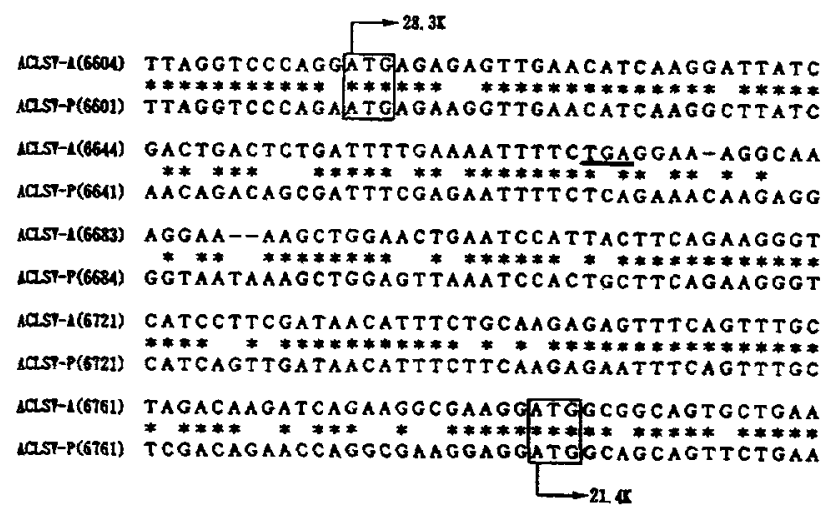

Fig. 3. Nucleotide sequence alignment of the regions surrounding the ORF 3 initiation codons of ACLSV-A and ACLSV-P. Asterisks indicate identical nucleotides in both isolates. The stop codon between the ATG and second ATG of ACLSV-A is underlined.

ORF 1 , preceded by a 151 base leader sequence, starts at AUG (positions 152 to 154) and ends at UGA (positions 5807 to 5809 ) and encodes a protein with an $M_{\mathrm{r}}$ of $216503(216 \cdot 5 \mathrm{~K})$. The ORF 1 protein contains two motifs associated with the helicase (GxxxGxGKS) and the RNA polymerase (GxxxTxxxNT, GDD) at positions 1060 to 1067 and 1694 to 1728 , respectively (Fig. 1). The overall similarity of the ORF 1 protein between ACLSV$A$ and ACLSV-P is $88.4 \%$ (Table 1). However, the regions surrounding the above two motifs were highly conserved between both isolates and their identities were 97.6\% (positions 1058 to 1305 for ACLSV-A and 1057 to 1304 for ACLSV-P) and $97.7 \%$ (positions 1637 to 1767 for ACLSV-A and 1636 to 1766 for ACLSV-P), respectively.

ORF 2 begins at AUG (positions 5727 to 5729) and terminates at UGA (positions 7098 to 7100 ) to yield a protein with an $M_{\mathrm{r}}$ of $50453(50 \cdot 4 \mathrm{~K})$ (Fig. 1). The

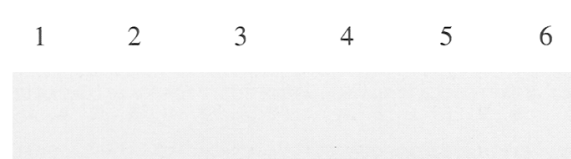

Fig. 4. Immunoblot analysis of the proteins expressed in $E$. coli containing pTrc99A-CP. Lane 1, proteins from $E$. coli cells containing pTrc99A grown in the presence of IPTG; lane 2, proteins from $E$. coli containing pTrc99A-CP without IPTG; lane 3, proteins from $E$. coli containing pTrc99A-CP induced by IPTG; lane 4, coat protein from purified ACLSV-A; lane 5, proteins from C. quinoa leaves infected with ACLSV-A; lane 6, proteins from healthy $C$. quinoa leaves. An arrowhead indicates the position of the coat protein of ACLSV-A.

position of the initiation codon of the $50 \cdot 4 \mathrm{~K}$ protein is six nt downstream from the corresponding position in ACLSV-P (Fig. 2). This location of the initiation codon was the same in the three different cDNA clones sequenced. The $50.4 \mathrm{~K}$ protein of ACLSV-A, as did that of ACLSV-P, showed an amino acid similarity of $22.4 \%$ with the gene I protein of cauliflower mosaic virus (Franck et al., 1980), and the identity of the ORF 2 protein between ACLSV-A and ACLSV-P was 79.9\% (Table 1).

ORF 3 contains an AUG (positions 6784 to 6786) and ends at UGA (positions 7363 to 7365 ) encoding a protein with an $M_{\mathrm{r}}$ of $21394(21.4 \mathrm{~K})$ which is dissimilar to the $28.3 \mathrm{~K}$ protein encoded by ORF 3 of ACLSV-P. This discrepancy is explained as follows. The initiation codon (AUG, positions 6616 to 6618) is located in the ACLSVA genome at the almost same position as the AUG codon of the $28.3 \mathrm{~K}$ protein of ACLSV-P, but the termination codon (UGA, positions 6670 to 6672) exists between the first AUG and the second AUG (positions 6784 to 6786 ) which is the initiation codon of the $21.4 \mathrm{~K}$ protein (Fig. 3). The identity of ORF 3 proteins between both isolates was $88.6 \%$ (Table 1 ).

In in vitro translation experiments reported previously 
(Yoshikawa \& Takahashi, 1989), ACLSV-A RNA directed the synthesis of $105 \mathrm{~K}$ and $51 \mathrm{~K}$ proteins as major products and several minor proteins of which the $23 \mathrm{~K}$ protein was immunoprecipitated with ACLSV-A antiserum. The products ( $51 \mathrm{~K}$ and $23 \mathrm{~K}$ proteins) detected in translation experiments may be synthesized from fragmented RNAs of ACLSV-A RNA because the proteins encoded by ORFs 2 and 3 are thought to be translated from subgenomic RNAs (German et al., 1992).

The $M_{\mathrm{r}}(21.4 \mathrm{~K})$ of the protein encoded by ORF 3 of ACLSV-A agrees with that of the ACLSV-A coat protein $M_{\mathrm{r}}$ estimated by PAGE (Yoshikawa \& Takahashi, 1988 ). To examine whether the $21.4 \mathrm{~K}$ protein is indeed the coat protein, the ORF 3 region was amplified by PCR using pBCLS38 containing the $3.8 \mathrm{~kb}$ insert of the 3 '-terminal region, a synthetic oligonucleotide (5' AAGAATTCAGGATGGCGGCAGTGCTG 3' corresponding to nt positions 6781 to 6798 and containing an EcoRI site) and a universal primer ( $5^{\prime}$ AACAGCTATGACCATG 3') located in the multicloning site of the BS plasmid. The PCR product was digested with EcoRI and KpnI and ligated to a pTrc99A expression vector restricted with the same enzymes (designated pTrc99A-CP) and the product was used to transform $E$. coli JM105. Proteins were then prepared from the cultures grown in the presence of IPTG and analysed by immunoblotting using ACLSV-A antiserum as described previously (Yoshikawa et al., 1992). Fig. 4 shows the result of immunoblot analysis, in which the protein expressed in E. coli containing pTrc99A-CP reacted with the antiserum against purified ACLSV-A. This protein is slightly larger than coat protein from purified virus and infected $C$. quinoa leaves because the expressed protein contains four additional amino acids derived from the vector plasmid and ACLSV-A cDNA. These results demonstrate that the $21.4 \mathrm{~K}$ protein encoded by ORF 3 is the coat protein of ACLSV-A, supporting the results obtained before following the determination of the partial amino acid sequence of the ACLSV-P coat protein (German et al., 1990).

At present, ACLSV is classified into the closterovirus group, based mainly on its particle structure (Francki et al., 1991). This group is composed of heterogeneous virus species with different particle lengths, transmission modes and tissue localization of the virus particles in infected material. The genome organization of ACLSV is quite different from that of the 3 -terminal genomic region of beet yellows virus (Agranovsky et al., 1991), the type member of the closterovirus group, indicating that ACLSV should be classified into a new virus group. Further investigations on the genome organization of closteroviruses will provide valuable information on the classification of this group.

This work was supported in part by a Grant-in-Aid from the Ministry of Education, Science and Culture, Japan.

\section{References}

Agranovsky, A. A., Boyko, V. P., Karasev, A. V., Lunina, N. A., Koonin, E. V. \& DolJA, V. V. (1991). Nucleotide sequence of the 3'terminal half of beet yellows closterovirus RNA genome: unique arrangement of eight virus genes. Journal of General Virology $\mathbf{7 2}$, $15-23$.

Deborde, D. C., Naeve, C. W., Herlocher, M. L. \& Maassab, H. F. (1986). Resolution of a common RNA sequencing ambiguity by terminal deoxynucleotidyl transferase. Analytical Biochemistry 157, 275-282.

Franck, A., Guilley, H., Jonard, G., Richards, K. \& Hirth, L. (1980). Nucleotide sequence of cauliflower mosaic virus DNA. Cell 21, $285-294$.

Francki, R. I. B., Fauquet, C. M., Knudson, D. L. \& Brown, F. (editors) (1991). Classification and Nomenclature of Viruses. Fifth Report of the International Committee on Taxonomy of Viruses. Archives of Virology Supplementum 2, pp. 1-450.

German, S., Candresse, T., Lanneau, M., Huet, C., Pernollet, J. C. \& DunEZ, J. (1990). Nucleotide sequence and genome organization of apple chlorotic leaf spot closterovirus. Virology 179, 104-112.

German, S., Candresse, T., Le Gall, O., Lanneau, M. \& Dunez, J. (1992). Analysis of the dsRNAs of apple chlorotic leaf spot virus. Journal of General Virology 73, 767-773.

Gubler, U. \& HoffmanN, B. J. (1983). A simple and very efficient method for generating cDNA libraries. Gene 25, 263-269.

HeNIKOFF, S. (1984). Unidirectional digestion with exonuclease III creates targeted breakpoints for DNA sequencing. Gene 28, 351-359.

LISTER, R. M. (1970). Apple chlorotic leaf spot virus. $C M I / A A B$ Descriptions of Plant Viruses, no. 30.

NÉMETH, M. (1986). Virus, Mycoplasma and Rickettsia Diseases of Fruit Trees. Akadémiai Kiadó.

SANGer, F., Nicklen, S. \& Coulson, A. R. (1977). DNA sequencing with chain-terminating inhibitors. Proceedings of the National Academy of Sciences, U.S.A. 74, 5463-5467.

YANASE, H. (1974). Studies on apple latent viruses in Japan. Bulletin of the Fruit Tree Research Station, Japan Series C1, 47-109.

Yoshikawa, N. \& Takahashi, T. (1988). Properties of RNAs and proteins of apple stem grooving and apple chlorotic leaf spot viruses. Journal of General Virology 69, 241-245.

Yoshikawa, N. \& TAKahaSHI, T. (1989). In vitro translation of apple chlorotic leaf spot virus RNA. Journal of General Virology 70, 3051-3054.

Yoshikawa, N., Kanno, Y., Sasaki, E. \& Takahashi, T. (1988). Properties of apple stem grooving and apple chlorotic leaf spot viruses and molecular cloning of their genomes. In Abstracts of 5 th International Congress of Plant Pathology, Kyoto, p. 45.

Yoshikawa, N., Sasaki, E., Kato, M. \& Takahashi, T. (1992). The nucleotide sequence of apple stem grooving capillovirus genome. Virology 191, 98-105.

(Received 8 March 1993; Accepted 29 April 1993) 